\title{
MicroRNA-552 promotes tumor cell proliferation and migration by directly targeting DACH1 via the Wnt/ $\beta$-catenin signaling pathway in colorectal cancer
}

\author{
JIA CAO $^{1 *}$, XIU-RUI YAN ${ }^{2 *}$, TING LIU ${ }^{2}$, XUE-BO HAN ${ }^{1}$, JING-JING YU $^{2,4}$, SHI-HAI LIU ${ }^{3}$ and LI-BIN WANG ${ }^{1,2,4}$ \\ ${ }^{1}$ Department of Medical Laboratory, Ningxia Medical University, Clinical Medicine College, Yinchuan, Ningxia 750004; \\ ${ }^{2}$ Stem Cell Institute, The General Hospital of Ningxia Medical University, Yinchuan, Ningxia 750004; ${ }^{3}$ Central Laboratory, \\ The Affiliated Hospital of Qingdao University, Animal Experimental Center, Qingdao, Shandong 266003; ${ }^{4}$ National \\ Engineering Research Center of Beijing Biochip Technology, Sub-Center in Ningxia, Yinchuan, Ningxia 750004, P.R. China
}

Received March 9, 2016; Accepted January 31, 2017

DOI: $10.3892 / \mathrm{ol} .2017 .6600$

\begin{abstract}
The purpose of the present study was to analyze the crucial role of microRNAs (miRNAs/miRs) involved in the proliferation and migration of colorectal cancer (CRC) and to investigate their underlying mechanisms. The present study discusses the expression and function of miR-552 in CRC. The expression level of miR-552 in CRC cells and tissues was observed, and it was suggested that the high expression of miR-552 accelerated the proliferation and migration of CRC cells in vitro. Notably, a result of the present study was that the cell fate determination factor Dachshund family transcription factor 1 (DACH1) was identified as a direct target of miR-552. Suppressing miR-552 expression in CRC cells increased endogenous DACH1 mRNA and protein levels, which was negatively correlated with miR-552. DACH1 performs an important role in the development of a number of neoplasms, and has the ability to regulate the $\mathrm{Wnt} / \beta$-catenin signaling pathway as a novel predictive and diagnostic biomarker. Accordingly, it was concluded that miR-552 exerted a tumor-promoting role in $\mathrm{CRC}$ development by targeting DACH1, which may contribute to the increase in the rates of CRC proliferation and migration. miR-552 may serve as a potential diagnostic and prognostic biomarker for CRC.
\end{abstract}

Correspondence to: Dr Shi-Hai Liu, Central Laboratory, The Affiliated Hospital of Qingdao University, Animal Experimental Center, 1677 Wutaishan Street, Qingdao, Shandong 266003, P.R. China E-mail: shliumed@126.com

Dr Li-Bin Wang, Department of Medical Laboratory, Ningxia Medical University, Clinical Medicine College, 804 Shengli Street, Yinchuan, Ningxia 750004 P.R China

E-mail: wanglibin007@126.com

*Contributed equally

Key words: microRNA-552, dachshund family transcription factor 1, colorectal cancer, proliferation, migration

\section{Introduction}

Colorectal cancer (CRC) has one of the highest cure rates of all types of malignant tumors (1), however remains ranked as the fourth leading cause of cancer-associated mortality in the world (2). In previous years, the morbidity and mortality rates of CRC have significantly increased due to an ageing population, and with changes in eating habits and lifestyles (3). The development of distant metastasis is a major cause of cancer-associated mortalities in CRC patients (4). Overwhelming evidence has demonstrated that aberrant expression of microRNA (miRNA/miR) contributes to CRC development by affecting the expression of the genes that regulate cancer progression (5).

miRNAs, endogenous small non-coding regulatory RNAs measuring 18-25 nucleotides long (6), usually regulate gene expression in a number of tumor-associated signaling pathways at the post-transcriptional level, including the $\mathrm{Wnt} / \beta$-catenin signaling pathway (7). As miRNAs tend to be localized to fragile chromosomal regions (8), they have the ability to adjust the levels of their corresponding mRNAs, and serve critical roles in the physiological and pathological processes of tumor development, which are a novel aspect of cancer studies. Previous evidence has demonstrated that miRNAs are involved in a number of biological processes, including proliferation, differentiation, migration, angiogenesis and protein splitting (9-11). miRNAs also serve as tumor promoter genes or tumor suppressor genes by negatively regulating their targets. These data suggest a possibility that miRNA is a novel focus for examining the current diagnosis and treatment of tumors.

Previously, several studies revealed that miR-124 results in a decrease in the proliferation ability of CRC cells by targeting ribose-phosphate pyrophosphokinase 1 and ribose 5-phosphate isomerase mRNAs (12), that miR-146a directs the symmetric division of colorectal cancer stem cells (13) and that miR-101 serves as an endogenous proteasome inhibitor that suppresses tumor cell proliferation via targeting of the proteasome assembly factor proteasome maturation protein (14). Therefore, attention has been focused on the activities of miRNA in cancer development. 
In the present study, it was demonstrated that the upregulation of miR-552 is observed in CRC cells and tissues. In vitro experiments revealed that the high expression of miR-552 was associated with CRC proliferation and migration. Notably, it was demonstrated that Dachshund family transcription factor 1 (DACH1) is a direct target of miR-552, the levels of which decreased when miR-552 was overexpressed. In vitro, the $\mathrm{Wnt} / \beta$-catenin signaling pathway is a key pathway for the proliferation of CRC cells (15). The restoration of normal expression levels of DACH1 affected the Wnt/ $\beta$-catenin signaling pathway and the corresponding downstream targets; therefore it may be possible that miR-552 contributes to tumor proliferation and migration via inhibiting DACH1 and serves as a marker for poorer prognoses. Therefore, it may be a potential target for therapeutic intervention in patients with CRC.

\section{Materials and methods}

Total RNA extraction and reverse transcription-quantitative polymerase chain reaction $(R T-q P C R)$. Total RNA was isolated from tissues samples and cell lines using TRIzol reagent (Takara Bio, Inc., Otsu, Japan) according to the manufacturer's protocol, which efficiently recovered all RNA species, including miRNAs. RNA quality and quantity were measured using a spectrophotometer and RNA integrity was determined by gel electrophoresis. cDNA was reverse-transcribed and amplified by PCR using Reverse Transcription kit (Takara Bio, Inc.) and miRNA Real-Time PCR assay kit (GeneCopoeia, Inc., Rockville, MD, USA). The expression level of miR-552 was measured using miR-552 specific primers and SYBR-Green fluorophore (Takara Bio, Inc.) probes. The primers used are listed in Table I. The reverse transcription product was $2 \mu \mathrm{l}$, and the PCR reaction system was as follows: 40 cycles of pre-denaturation at $95^{\circ} \mathrm{C}$ for $10 \mathrm{~min}$, followed by annealing and stretching at $95^{\circ} \mathrm{C}$ for $15 \mathrm{sec}$ and $60^{\circ} \mathrm{C}$ for $1 \mathrm{~min}$, respectively. U6 and GAPDH were used as internal controls for miR-552 and DACH1, respectively. The relative expression of each gene was quantified by the $2^{-\Delta \Delta \mathrm{Cq}}$ method (16). The experiment was performed in triplicates.

Clinical samples, cell lines, in vitro culture and transfection. The present study was approved by the Ethics Review Committees of the General Hospital of Ningxia Medical University, (Yinchuan, Ningxia, China), with written informed consent being obtained from all patients. A total of 20 pairs of CRC tissues and matched adjacent normal tissues were collected from patients during surgical resection. LOVO, SW620, HCT116 and NCM460 cells were all purchased from the Cell Bank of Type Culture Collection of Chinese Academy of Sciences (Shanghai, China). Cells were cultured in RPMI-1640 medium (Gibco; Thermo Fisher Scientific, Inc., Waltham, MA, USA) supplemented with $10 \%$ fetal bovine serum (FBS) and $1 \%$ antibiotics at $37^{\circ} \mathrm{C}$ at $5 \% \mathrm{CO}_{2}$. The cells were transfected with $20 \mu \mathrm{M}$ miR-552 inhibitor (miR-552-in) or miR-552-inhibitor negative control (NC) at $\sim 50 \%$ density using Effectene transfection reagent (Invitrogen; Thermo Fisher Scientific, Inc.) according to the manufacturer's protocol.
Table I. Reverse transcription-quantitative polymerase chain reaction primers.

\begin{tabular}{ll}
\hline Gene & \multicolumn{1}{c}{ Sequence $\left(5^{\prime} \rightarrow 3^{\prime}\right)$} \\
\hline miR-552 & AACAGGTGACTGGTTAGACAA \\
U6 & ATTGGAACGATACAGAGAAGATT \\
Forward & \\
Reverse & CGAACGCTTCACGAATTTG \\
DACH1 & \\
Forward & CCCTCTACAATGACTGCACCA \\
Reverse & GCGGCATGATGTGAGAGTTCT \\
c-Myc & \\
Forward & GTCAAGAGGCGAACACACAAC \\
Reverse & TTGGACGGACAGGATGTATGC \\
Cyclin D1 & \\
Forward & GTCTGTGCATTTCTGGTTGCA \\
Reverse & TTTCTAGACTTTCATGTTTGTCTTTTTGTC \\
GAPDH & \\
Forward & TCATGGGTGTGAACCATGAGAA \\
Reverse & GGCATGGACTGTGGTCATGAG \\
GSK3 3 & \\
Forward & AGACGCTCCCTGTGATTTATGT \\
Reverse & CCGATGGCAGATTCCAAAGG \\
$\beta$-catenin & \\
Forward & ATGTCCAGCGTTTGGCTGAA \\
Reverse & TGGTCCTCGTCATTTAGCAGTT \\
\hline
\end{tabular}

miR, microRNA; DACH1, Dachshund family transcription factor 1; GSK3 $\beta$, glycogen synthase kinase $3 \beta$.

miR-552 target gene prediction. Extensive experiments and comprehensive studies have demonstrated that miRNAs, negative regulators of target genes, express its biological function directly or indirectly (17). In this study, the putative miR-552 target genes were predicted using MiRanda (Memorial Sloan-Kettering Cancer Center, New York, NY, USA), PicTar (Computational Medicine Center, Philadelphia, PA, USA) and TargetScan (Whitehead Institute for Biomedical Research, Cambridge, MA, USA) software. Followed the procedure of Betel et al (18), the present study identified DACH1 as a target gene of miR-552.

Luciferase reporter assay. A total of $\sim 1 \times 10^{4}$ cells were seeded in a 96-well plate and were co-transfected miR-552-in and wild type (Wt) or mutant (Mut) DACH1 reporter plasmids (Jikai Gene Chemical Co., Ltd., Shanghai, China) into LOVO and SW620 cells using Effectene transfection reagent. The cells were washed with PBS and harvested after $48 \mathrm{~h}$. Luciferase activity was measured with dual-luciferase reporter assay system (Promega Corporation, Madison, WI, USA).

Cell proliferation assay. For the colony formation assay, LOVO and SW620 cells were grown in 6-well culture plates and transfected with miR-552-in or NC using Effectene transfection reagent. After 7-10 days, the cells were fixed with 
A

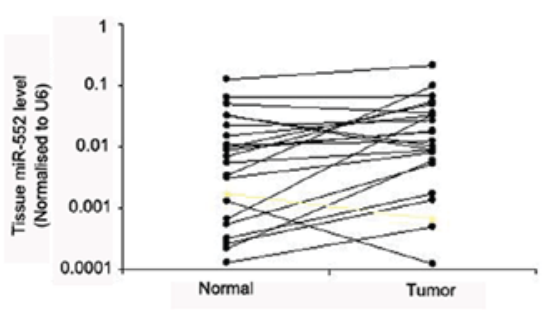

B

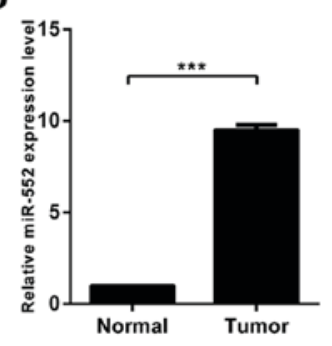

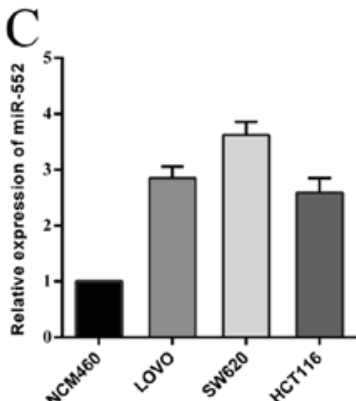

Figure 1. miR-552 is upregulated in CRC tissues and cell lines. (A) The expression of miR-552 in 20 pairs of CRC tissues and adjacent non-tumorous colorectal tissues were examined by RT-qPCR. (B) The average value of miR-552 expression in tumor tissues and normal tissues. (C) The expression levels of miR-552 in the normal colorectal NCM460 cell line and three CRC cell lines; LOVO, SW620 and HCT116. Values are presented as the mean \pm standard deviation from three experiments. ${ }^{* * *} \mathrm{P}<0.001$. miR, microRNA; CRC, colorectal cancer; RT-qPCR, reverse transcription-quantitative polymerase chain reaction.

methanol for $15 \mathrm{~min}$ and stained with $0.2 \%$ crystal violet for visualization and counting. For the MTT assay, $100 \mu \mathrm{l}$ cells per well were seeded into 96-well plates, and were transfected with miR-552-in or the negative control (miR-552-inhibitor-NC) using Effectene transfection reagent. The cell proliferation rate was detected at 24, 48, 72 and $96 \mathrm{~h}$. The cells were disposed with $20 \mu \mathrm{l}$ MTT solution and incubated at $37^{\circ} \mathrm{C}$ for $4 \mathrm{~h}$. Subsequently, $150 \mu 1$ dimethyl sulfoxide was added and the absorbance values were detected using Thermo Multiskan Go Microplate Reader (Thermo Fisher Scientific, Inc.) at $490 \mathrm{~nm}$. The NC group was the control.

Wound-healing assay. Approximately $70 \%$ of the cell density was planted in 12-well plate prior to transfection. The wound-healing assay was implemented to determine the cell migration ability subsequent to transfection with miR-552-in or $\mathrm{NC}$, and the cell migration rate was recorded by microscopy at 0 and $24 \mathrm{~h}$ when the monolayer was at $90 \%$ density.

Transwell assay. The cells were grown in 24-well culture plates and transfected with miR-552-in or NC using Effectene transfection reagent. Cells were harvested after $48 \mathrm{~h}$. The Transwell assay was performed using $8-\mu \mathrm{m}$ pore size Corning chambers (Corning Costar, Corning, NY, USA). The lower chamber was filled with $600 \mu \mathrm{l}$ RPMI-1640 medium containing 20\% FBS and Recombinant Human platelet-derived growth factor-BB (Invitrogen; Thermo Fisher Scientific, Inc.) was added. The Matrigel-coated insert (BD Biosciences, Franklin Lakes, NJ, USA) was placed and cells were counted to $15 \times 10^{4}$ cells in the upper chamber. The non-migrated or non-invaded cells were gently removed from the upper surface of the membrane by cotton swab, and the migrated cells were attached to the lower surface after $24 \mathrm{~h}$. The cells were fixed with $100 \%$ methanol and stained using $0.2 \%$ crystal violet solution for $15 \mathrm{~min}$. Cells that migrated onto the lower surface were counted using a CKX-41 inverted fluorescence microscope with magnification, x100 (Olympus Corporation, Tokyo, Japan) in five randomly chosen visual fields.

Western blotting. The cells were lysed in protein extraction radioimmunoprecipitation buffer $[20 \mathrm{mM}$ Tris- $\mathrm{HCl}(\mathrm{pH} 7.4)$, $100 \mathrm{mM} \mathrm{NaCl}, 10 \% \mathrm{NP}-40,10 \%$ sodium deoxycholate and $100 \mathrm{mM}$ EDTA] and each sample was determined using a bicinchonic acid protein assay reagent kit (Thermo Fisher
Scientific, Inc.) subsequent to transfection with miR-552-in or $\mathrm{NC}$ for $48 \mathrm{~h}$. A total of $20 \mu \mathrm{g}$ of lysates were electrophoresed by $10 \%$ SDS-PAGE and transferred onto $0.45-\mu \mathrm{m}$ polyvinylidene fluoride membranes (Merck KGaA, Darmstadt, Germany). Subsequently, the proteins were blocked with $5 \%$ non-fat milk for $1 \mathrm{~h}$ at room temperature. The membranes were incubated with primary antibodies, including: Anti cyclin D1 (rabbit monoclonal; dilution, 1:1,000; cat no. 2926), anti c-myc (rabbit monoclonal; dilution, 1:1,000; cat no. 5605), anti $\beta$-catenin (rabbit monoclonal; dilution, 1:500; cat no. 8480); anti GSK3 $\beta$ (rabbit monoclonal; dilution 1:1,000; cat no. 12456S) (all from Cell Signaling Technology, Inc., Danvers, MA, USA); anti DACH1 (rabbit monoclonal; dilution 1:1,000; cat no. 10914-1-A P; Wuxi AccoBio BioTech Inc., Wuxi, China), GAPDH (mouse monoclonal; dilution, 1:5,000; cat no. 2D4A7; Novus Biologicals, LLC., Littleton, CO, USA) overnight at $4^{\circ} \mathrm{C}$, followed by incubation with the horseradish peroxidase conjugated secondary antibodies (anti-rabbit: dilution, 1:10,000; cat no. \#7074; or anti-mouse: dilution, 1:10,000; cat no. \#7076) (both from Cell Signaling Technology, Inc.) for $1.5 \mathrm{~h}$ at room temperature. The protein expression was normalized against GAPDH and grayscale analysis was performed using Image-Pro plus (version 6.0) software (Media Cybernetics, Rockville, MD, USA). The experiment was performed in triplicate.

Statistical analysis. All statistical analyses were performed using the SPSS 17.0 statistical software package (SPPS, Inc. Chicago, IL, USA) or GraphPad Prism 6.0 (GraphPad Software, Inc., La Jolla, CA, USA). The difference between two groups was determined as mean \pm standard deviation and statistical significance was analyzed using a t-test or one-way analysis of variance followed by Bonferroni's multiple comparison test. $\mathrm{P}<0.05$ was considered to indicate a statistically significant difference.

\section{Results}

miR-552 is upregulated in CRC tissues and cell lines. In order to confirm the involvement of miR-552 in CRC carcinogenesis, the expression of miR-552 was measured by RT-qPCR in 20 pairs of $\mathrm{CRC}$ tissues and their adjacent normal tissues. The results indicated that the expression level of miR-552 in CRC tissues was significantly higher compared with that in matched normal tissues (Fig. 1A and B). It was also demonstrated that 
A

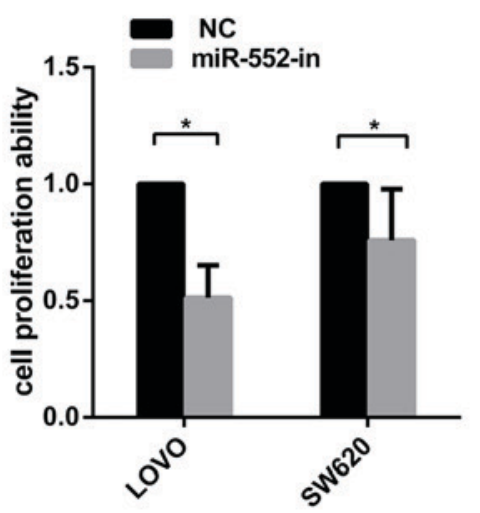

B

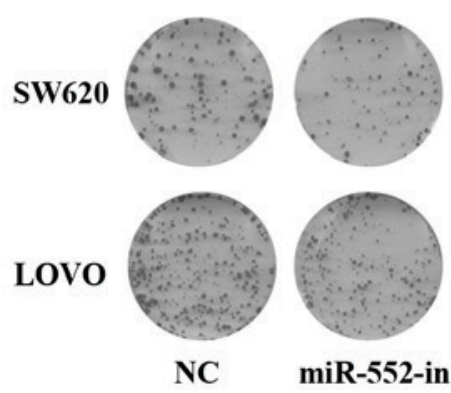

C

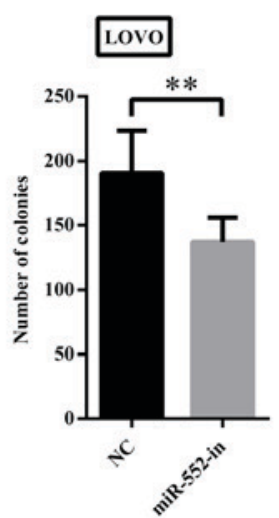

D

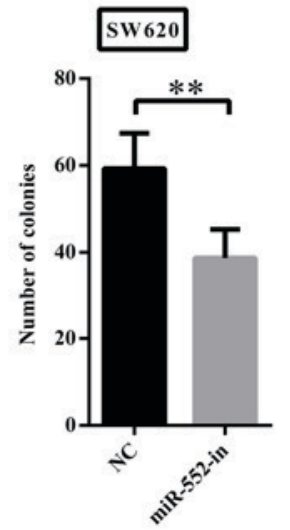

Figure 2. Ectopic expression of miR-552 promotes colorectal cell proliferation in vitro. (A) Results of an MTT assay demonstrating that cellular proliferation was inhibited by transfection with miR-552-in. (B) Effects of miR-552-in on cell proliferation in vitro by plate colony formation assay. Ectopic miR-552 expression inhibits cell proliferation in (C) LOVO and (D) SW620 cells. ${ }^{* *} \mathrm{P}<0.01$. miR, microRNA; miR-552-in, miR-552 inhibitor; NC, miR-552-inhibitor-NC.

miR-552 was upregulated in the CRC LOVO, SW620 and HCT116 cell lines, when compared with normal colorectal NCM460 cell line (Fig. 1C), which is consistent with the results identified in CRC tissues. In conclusion, these observations suggest that the overexpression of miR-552 may serve important roles in CRC carcinogenesis and progression.

Ectopic expression of miR-552 promoted CRC cell proliferation in vitro. The increased expression of miR-552 in CRC cell lines suggested that miR-552 may be an oncogene. To additionally investigate the biological functions of miR-552 in CRC development, MTT and colony formation assays were performed to assess the role of miR-552 in CRC cell viability and proliferation. The results of the MTT assay illustrate that miR-552-in significantly reduced the viability of CRC cells compared with the control group (Fig. 2A). The colony formation assay also revealed that the overexpression of miR-552 increased the proliferation ability in the two CRC cell lines (Fig. 2B). The inhibition efficiency was additionally measured by calculating the entire field of vision on the number of cells (Fig. 2C and D).

Overexpression of $\mathrm{miR}-552$ promotes migration of $C R C$ cell in vitro. To determine whether overexpression of miR-552 exhibited a crucial role in migration and invasion, wound-healing and Transwell assays were performed. Fig. 3A and B illustrates that decreased expression of miR-552 (miR-552-in) was associated with significantly slower wound closure in LOVO and SW620 cells compared with their corresponding controls. Secondly, the Transwell assay also demonstrated that transfection with miR-552-in exhibited lower migratory and invasive activities compared with the control groups (Fig. 3C). In addition, the number of miR-552-in transfected migrating cells was calculated using statistical software (Fig. 3D). Thus, it is suggested that miR-552 clearly facilitates CRC cell migration in vitro.

DACH1 is a direct target of miR-552 in CRC cells. DACH1 is frequently downregulated in CRC and is closely associated with poorer prognoses (19). Based on MiRanda software, it was predicted that DACH1 may be a potential downstream target of miR-552. To explore whether DACH1 is a direct target of miR-552 in the CRC cellular environment, a dual-luciferase reporter assay was performed. miR-552-in or NC with plasmids containing 3'untranslated regions (UTR) of wt-DACH1 or mut-DACH1 were transfected into LOVO and SW620 cell lines. The results demonstrated that the downregulation of miR-552 significantly increased the relative luciferase activity of wt-DACH1-3'UTR in the two cell lines. However, no difference in luciferase activity was observed in mut-DACH1-3'UT R (Fig. 4A and B).

miR-552 negatively regulates DACH1 expression at the post-transcriptional level. The LOVO and SW620 cells were transfected with miR-552-in or NC to measure transfection efficiency (Fig. 5A) and to verify the expression of DACH1 mRNA and protein levels. It was revealed with RT-qPCR (Fig. 5B) and western blot analysis (Fig. 5C) that miR-552-in markedly increased the DACH1 mRNA and protein expression levels.

miR-552 affects the Wnt/ $\beta$-catenin signaling pathway. To investigate the underlying mechanism of miR-552 in CRC, the biological functions of the genes associated with the Wnt/ $\beta$-catenin signaling pathway were analyzed. Western blotting was used to explore gene expression of the Wnt/ $\beta$-catenin signaling pathway at the protein level. The results demonstrated that an abnormal expression of DACH1 significantly inhibited the abundance of c-Myc, and that the higher expression of DACH1 was usually accompanied by a lowered expression of c-Myc (Fig. 6A). DACH1 was dramatically upregulated in response to miR-552-in. By contrast, the expression of c-Myc was decreased (Fig. 6B). In addition, other members of the Wnt/ $\beta$-catenin signaling pathway including glycogen synthase kinase $3 \beta(\mathrm{GSK} 3 \beta)$, cyclin D1 and $\beta$-catenin were examined via western blotting subsequent to transfection. The results demonstrated that miR-552-in inhibited the expression of cyclin D1 and c-Myc without altering the expression of GSK $3 \beta$ and $\beta$-catenin when compared with the corresponding negative controls (Fig. 6C). The average relative gray value of 
A

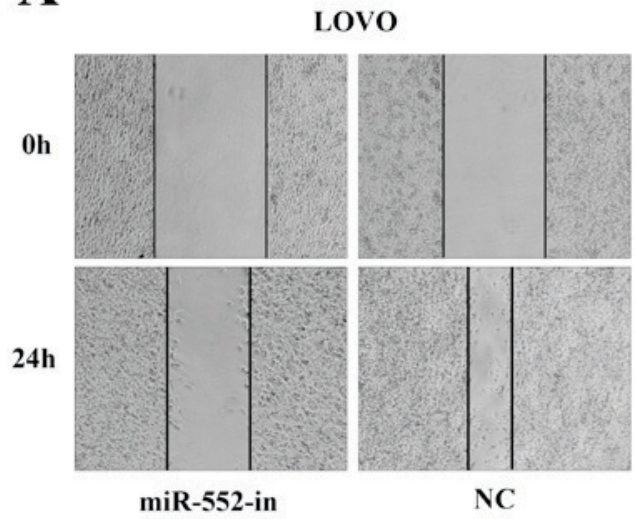

C

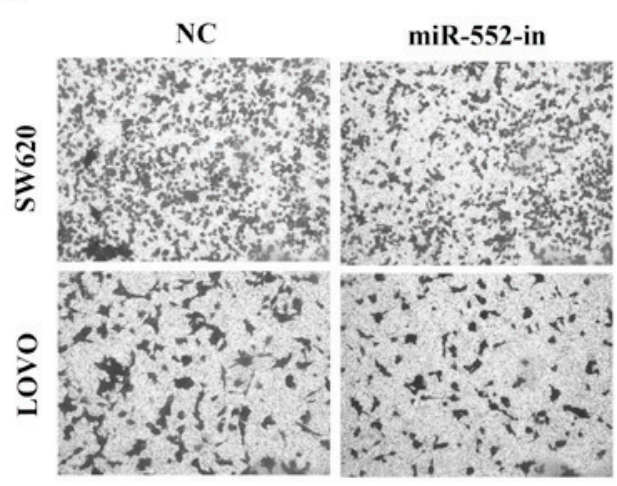

B

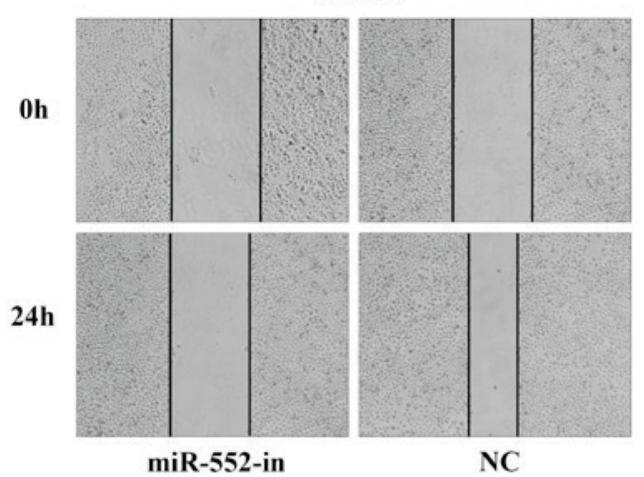

D
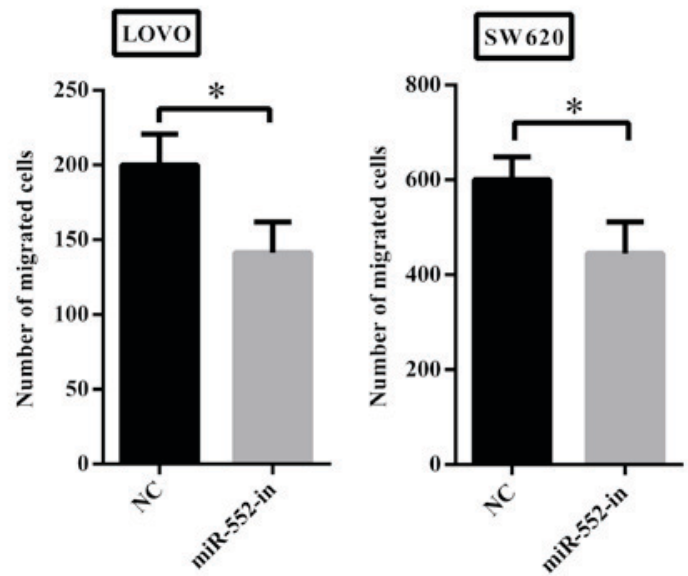

Figure 3. Effects of miR-552 on cell migration of colorectal cancer cells in vitro. Cell motility was evaluated by wound healing assay. At least three independent experiments for (A) LOVO and (B) SW620 cells were performed (magnification, x40). Cell migration was evaluated by (C) Transwell assay (magnification, $\mathrm{x} 100$ ), and by calculation of the migration rate of the total cell number. (D) Quantification of the migration assay demonstrating that miR-552-in suppressed the migration ability of LOVO and SW620 cells. "P<0.05. The data are expressed as the mean \pm standard deviation of three independent experiments. miR, microRNA; miR-552-in, miR-552 inhibitor; NC, miR-552-inhibitor-NC.

A

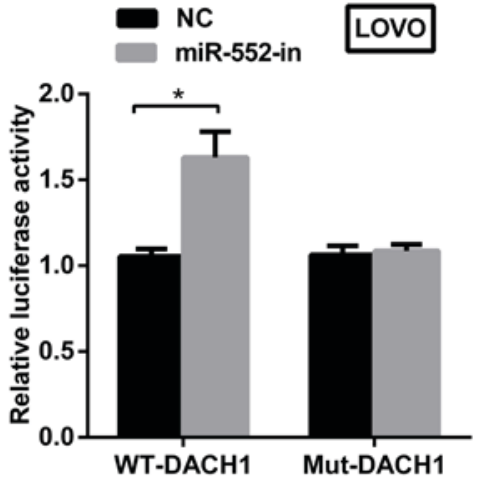

B

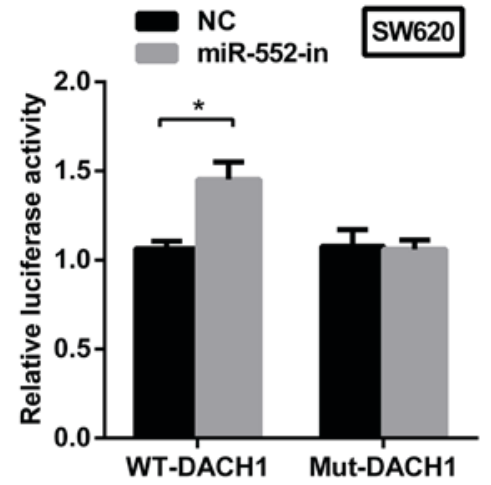

Figure 4. DACH1 is a direct target of miR-552 in colorectal cancer cells. The pcDNAmiR-552 expression plasmid or the negative control, containing wild-type and mutant-type 3'untranslated region-DACH1, were co-transfected into LOVO and SW620 cells. Luciferase activities were measured in (A) LOVO and (B) SW620 cells. "P<0.05. DACH1, Dachshund family transcription factor 1; miR, microRNA; WT, wild type; MUT, mutant; miR-552-in, microRNA-552 inhibitor; NC, miR-552-inhibitor-NC.

DACH1 and c-Myc are also presented (Fig. 6D). In conclusion, miR-552 promotes proliferation and migration of CRC and activates the Wnt/ $\beta$-catenin signaling pathway by directly regulating corresponding target proteins.

\section{Discussion}

The molecular mechanisms of proliferation and migration in CRC requires additional study in vivo and in vitro (20). Data 
A

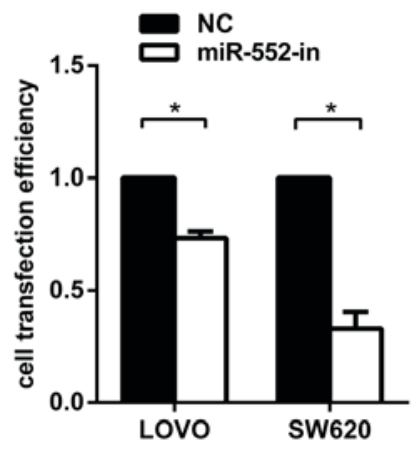

B

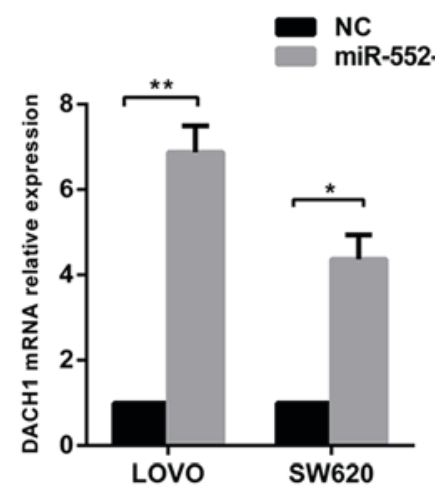

C

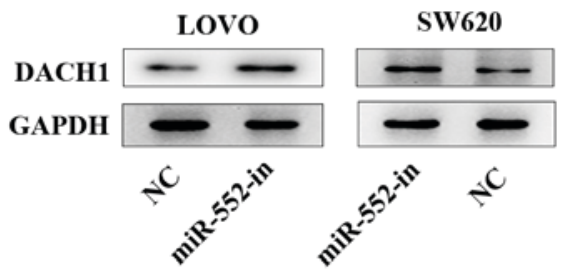

Figure 5. miR-552 negatively regulates DACH1 expression at the post-transcriptional level. (A) The transfection efficiency of reagents was detected when LOVO and SW620 cells were transfected with miR-552-in and NC. (B) Transfection of miR-552-in led to an increase in the expression level of DACH1 mRNA in LOVO and SW620 cells when compared with the corresponding negative controls. (C) Representative western blot analysis of DACH1 in LOVO and SW620 cells when transfected with miR-552-in. "P<0.05, ${ }^{* *} \mathrm{P}<0.01$. miR, microRNA; miR-552-in, miR-552 inhibitor; NC, miR-552-inhibitor-NC; DACH1, Dachshund family transcription factor 1 .
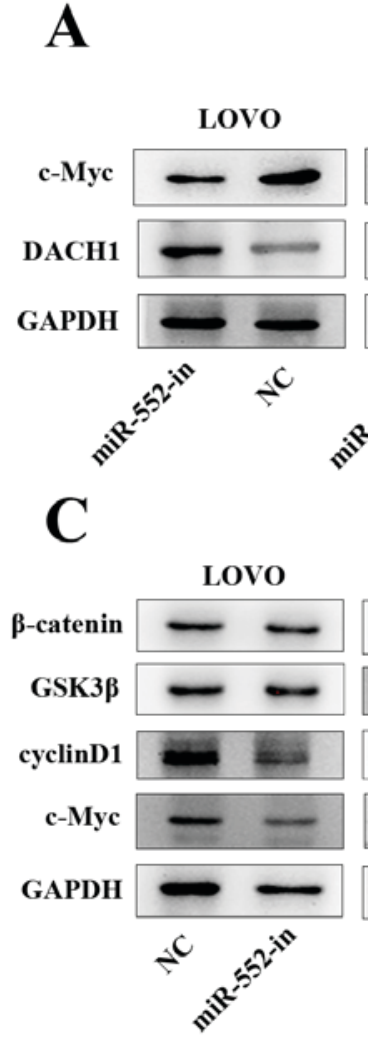

SW620

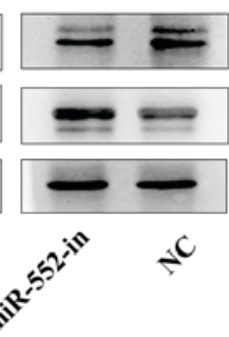

SW620

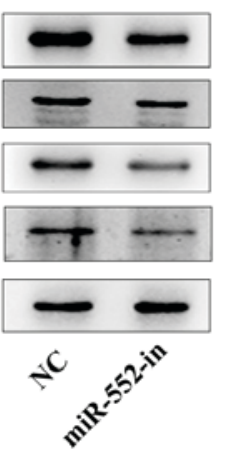

B

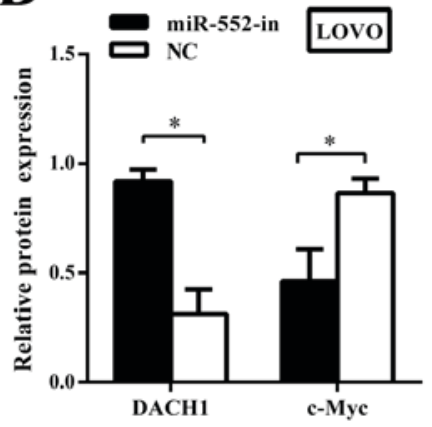

D

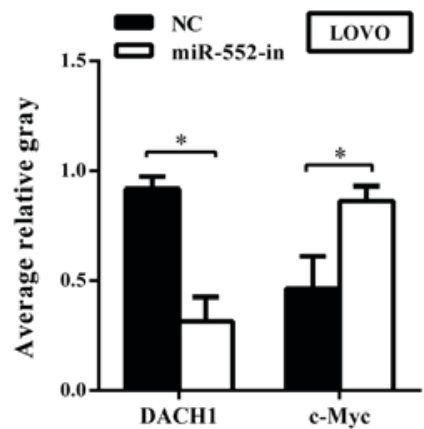

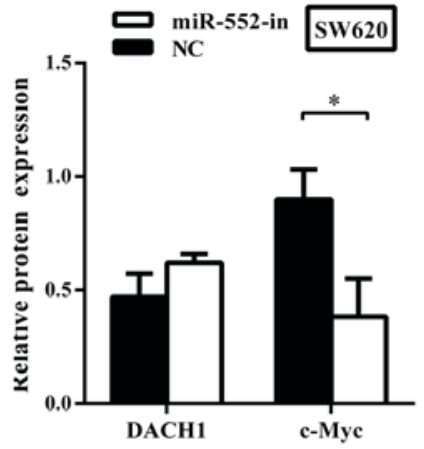

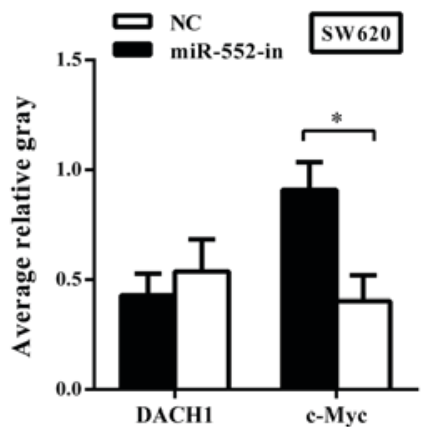

Figure 6. miR-552 affects the Wnt/ $\beta$-catenin signaling pathway. (A) Representative western blotting analysis of DACH1 and c-Myc in LOVO and SW620 cells (B) Expression levels of DACH1 and c-Myc proteins in LOVO and SW620 cells when treated with miR-552-in or NC vector. (C) Effects of miR-552-in on proteins of the Wnt/B-catenin signaling pathway. (D) The average relative gray of DACH1 and c-Myc from (A) were detected in colorectal cancer cell lines when treated with miR-552-in or NC vector. "P<0.05. miR, microRNA; miR-552-in, miR-552 inhibitor; NC, miR-552-inhibitor-NC; DACH1, Dachshund family transcription factor 1.

from several previous studies have indicated that the interactions between miRNAs and their corresponding mRNA regulate the expression of proto-oncogenes or tumor suppressor genes (21) and determine the malignant characteristics of tumor cells. Therefore, a comprehensive understanding of the association between miRNAs and tumor development is crucial for the diagnosis and treatment of cancer (22). In the present study, the expression level of miR-552 was initially measured in CRC cells and tissues, and the results demonstrated that the expression of miR-552 was significantly increased in the CRC cells and tissues compared with that in normal cells and tissues. Notably, these data indicated that a high expression 
of miR-552 was clearly associated with the proliferation and migration of CRC. The present study may explain the function of miR-552 in CRC.

It has been demonstrated that miR-552 is overexpressed in CRC cells and tissues (23). Additionally, the expression of miR-552 is markedly upregulated in the side population (SP) of CRC cells, which is closely associated to the resistance to multiple chemotherapeutics demonstrated in CRC (24). Although the importance of miR-552 in CRC has received attention in previous years, the hypothesis that miR-552 promotes the proliferation and migration of CRC has not been demonstrated. A notable aspect of the present study was that miR-552 facilitated CRC cell proliferation and migration in vitro using the colony formation and MTT assays to detect CRC cell proliferation ability. The wound-healing and Transwell assays were performed to test migration ability of CRC. The results demonstrated that an overexpression of miR-552 markedly promoted the proliferation and migration capabilities of CRC cells. Taken together, these data highlight the function of miR-552 as a tumor promoter in the progression of CRC.

As a cancer suppress gene, DACH1 is a crucial member of the Retinal Determination Gene Network (25). Generally, it has been demonstrated to be downregulated in a wide number of tumors, such as breast, endometrial and prostate cancer (19). Additionally, DACH1 has been identified to inhibit transforming growth factor- $\beta$ signaling pathway in breast and ovarian cancer (26) and to restrain the Wnt/ $\beta$-catenin signaling pathway in CRC (27). However, the epigenetic regulation and mechanisms of DACH1 remain unclear. In the present study, it was revealed that DACH1 was a direct functional target of miR-552 in CRC cells. Firstly, the miR-552-dependent promotion of CRC cell proliferation and migration may be completely restored by DACH1 overexpression. Secondly, the upregulation of miR-552 leads to the downregulation of its target, DACH1, which in turn leads to proliferation and migration in CRC. Thirdly, miR-552 overexpression markedly reduced DACH1 mRNA and protein levels. Conversely, inhibition of the expression of miR-552 may significantly increase the activity of luciferase reporter containing the 3'UTR sequence of DACH1. All these data demonstrate that miR-552 exhibits a negative correlation with DACH1.

The Wnt/ $\beta$-catenin pathway is an evolutionarily conserved pathway, which is important in initiating and regulating a diverse range of cellular activities, including cell proliferation, calcium homeostasis and cell polarity (28). There are a number of target proteins in the $\mathrm{Wnt} / \beta$-catenin signaling pathway, including c-Myc, cyclin D1, MMP3 and LEF. Previous studies have revealed that DACH1 exhibited an inverse correlation with c-Myc and cyclin D1 (19). In combination with the results from the present study, it is hypothesized that miR-552 may have specific effects on the Wnt/ $\beta$-catenin signal pathway. To investigate these effects of miR-552 on the Wnt/ $\beta$-catenin pathway, DACH1, c-Myc and cyclin D1 protein expression levels were measured using western blot analysis. The results demonstrated that the restoration of expression levels of DACH1 may reduce the expression of $\mathrm{c}-\mathrm{Myc}$ and cyclin D1. As DACH1 is associated with the Wnt/ $\beta$-catenin pathway, and c-Myc and cyclin D1 are major downstream targets of the Wnt/ $\beta$-catenin signaling pathway, the results of this experiment suggested that miR-552 regulates the expression of DACH1 and c-Myc, therefore affecting the $\mathrm{Wnt} / \beta$-catenin signaling pathway in CRC.

In conclusion, the data of the present study demonstrates that miR-552 is significantly upregulated in CRC. This miRNA can potently promote CRC cell proliferation and migration in vitro by mediating $\mathrm{DACH} 1$, and activating the $\mathrm{Wnt} / \beta$-catenin signaling pathway by directly regulating the corresponding target proteins. The identification of tumor-specific miRNAs and their targets is crucial for the comprehensive understanding of CRC progression and development. These results suggest that miR-552 may be a predictive biomarker and a novel therapeutic target for patients with advanced CRC, and may improve the prognosis of all patients.

\section{Acknowledgements}

The present study was supported in part by grants from the Special Personnel Project of Ningxia Medical University (grant no. XT201414), and from the National Natural Science Foundations of China (grant no. 81560474). The authors would like to thank the Affiliated Hospital of Qingdao University for providing technical support.

\section{References}

1. Chen MB, Wei MX, Han JY, Wu XY, Li C, Wang J, Shen W and Lu PH: MicroRNA-451 regulates AMPK/mTORC1 signaling and fascin1 expression in HT-29 colorectal cancer. Cell Signal 26: 102-109, 2014.

2. Dong Y, Yu J and Ng SS: MicroRNA dysregulation as a prognostic biomarker in colorectal cancer. Cancer Manag Res 6: 405-422, 2014.

3. Gellad ZF and Provenzale D: Colorectal cancer: National and international perspective on the burden of disease and public health impact. Gastroenterology 138: 2177-2190, 2010.

4. Cristóbal I, Caramés C, Madoz-Gúrpide J, Rojo F, Aguilera O and García-Foncillas J: Downregulation of miR-214 is specific of liver metastasis in colorectal cancer and could play a role determining the metastatic niche. Int J Colorectal Dis 29: 885, 2014.

5. Bonfrate L, Altomare DF, Di Lena M, Travaglio E, Rotelli MT, De Luca A and Portincasa P: MicroRNA in colorectal cancer: New perspectives for diagnosis, prognosis and treatment. J Gastrointestin Liver Dis 22: 311-320, 2013.

6. Giordano S and Columbano A: MicroRNAs: New tools for diagnosis, prognosis, and therapy in hepatocellular carcinoma? Hepatology 57: 840-847, 2013.

7. Hur K, Toiyama Y, Okugawa Y, Ide S, Imaoka H, Boland CR and Goel A: Circulating microRNA-203 predicts prognosis and metastasis in human colorectal cancer. Gut 66: 654-665, 2017.

8. Croce CM: Causes and consequences of microRNA dysregulation in cancer. Nat Rev Genet 10: 704-714, 2009.

9. BaiR, Weng C, Dong H,LiS, Chen G and XuZ: MicroRNA-409-3p suppresses colorectal cancer invasion and metastasis partly by targeting GAB1 expression. Int J Cancer 137: 2310-2322, 2015.

10. Jinushi T, Shibayama Y, Kinoshita I, Oizumi S, Jinushi M, Aota T, Takahashi T, Horita S, Dosaka-Akita H and Iseki K: Low expression levels of microRNA-124-5p correlated with poor prognosis in colorectal cancer via targeting of SMC4. Cancer Med 3: 1544-1552, 2014.

11. Rokavec M, Öner MG, Li H, Jackstadt R, Jiang L, Lodygin D, Kaller M, Horst D, Ziegler PK, Schwitalla S, et al: IL-6R/STAT3/miR-34a feedback loop promotes EMT-mediated colorectal cancer invasion and metastasis. J Clin Invest 124: 1853-1867, 2014.

12. Qiu Z, Guo W, Wang Q, Chen Z, Huang S, Zhao F, Yao M, Zhao Y and He X: MicroRNA-124 reduces the pentose phosphate pathway and proliferation by targeting PRPS1 and RPIA mRNAs in human colorectal cancer cells. Gastroenterology 149: 1587-1598.e11, 2015 . 
13. Hwang WL, Jiang JK, Yang SH, Huang TS, Lan HY, Teng HW, Yang CY, Tsai YP, Lin CH, Wang HW and Yang MH: MicroRNA-146a directs the symmetric division of Snail-dominant colorectal cancer stem cells. Nat Cell Biol 16: 268-280, 2014.

14. Zhang X, Schulz R, Edmunds S, Krüger E, Markert E, Gaedcke J, Cormet-Boyaka E, Ghadimi M, Beissbarth T, Levine AJ, et al: MicroRNA-101 suppresses tumor cell proliferation by acting as an endogenous proteasome inhibitor via targeting the proteasome assembly factor POMP. Mol Cell 59: 243-257, 2015.

15. Slaby O, Svoboda M, Michalek J and Vyzula R: MicroRNAs in colorectal cancer: Translation of molecular biology into clinical application. Mol Cancer 8: 102, 2009.

16. Livak KJ and Schmittgen TD: Analysis of relative gene expression data using real-time quantitative PCR and the 2(-Delta Delta C(T)) Method. Methods 25: 402-408, 2001.

17. Della Vittoria Scarpati G, Calura E, Di Marino M, Romualdi C, Beltrame L, Malapelle U, Troncone G, De Stefano A, Pepe S, De Placido S, et al: Analysis of differential miRNA expression in primary tumor and stroma of colorectal cancer patients. Biomed Res Int 2014: 840921, 2014.

18. Betel D, Wilson M, Gabow A, Marks DS and Sander C: The microRNA.org resource: Targets and expression. Nucleic Acids Res 36: D149-D153, 2008.

19. Yan W, Wu K, Herman JG, Brock MV, Fuks F, Yang L, Zhu H, Li Y, Yang Y and Guo M: Epigenetic regulation of DACH1, a nove Wnt signaling component in colorectal cancer. Epigenetics 8: 1373-1383, 2013.

20. Liu Y, Zhou R, Yuan X, Han N, Zhou S, Xu H, Guo M, Yu S, Zhang $\mathrm{C}$, Yin T and Wu K: DACH1 is a novel predictive and prognostic biomarker in hepatocellular carcinoma as a negative regulator of $\mathrm{Wnt} / \beta$-catenin signaling. Oncotarget 6: 8621-8634, 2015.
21. Amirkhah R, Schmitz U, Linnebacher M, Wolkenhauer O and Farazmand A: MicroRNA-mRNA interactions in colorectal cancer and their role in tumor progression. Genes Chromosomes Cancer 54: 129-141, 2015.

22. Shen G, Rong X, Zhao J, Yang X, Li H, Jiang H, Zhou Q, Ji T, Huang S, Zhang J and Jia H: MicroRNA-105 suppresses cell proliferation and inhibits PI3K/AKT signaling in human hepatocellular carcinoma. Carcinogenesis 35: 2748-2755, 2014.

23. Kim J, Lim NJ, Jang SG, Kim HK and Lee GK: miR-592 and miR-552 can distinguish between primary lung adenocarcinoma and colorectal cancer metastases in the lung. Anticancer Res 34 : 2297-2302, 2014.

24. Xia ZS, Wang L, Yu T, Zhong W, Lian GD, Wu D, Zhou HM and Chen GC: MiR-5000-3p, miR-5009-3P and miR-552: Potential microRNA biomarkers of side population cells in colon cancer. Oncol Rep 32: 589-596, 2014

25. Atkins M, Jiang Y, Sansores-Garcia L, Jusiak B, Halder G and Mardon G: Dynamic rewiring of the Drosophila retinal determination network switches its function from selector to differentiation. PLoS Genet 9: e1003731, 2013.

26. Wang P: Suppression of DACH1 promotes migration and invasion of colorectal cancer via activating TGF- $\beta$-mediated epithelial-mesenchymal transition. Biochem Biophys Res Commun 460: 314-319, 2015

27. Chu Q, Han N, Yuan X, Nie X, Wu H, Chen Y, Guo M, Yu S and Wu K: DACH1 inhibits cyclin D1 expression, cellular proliferation and tumor growth of renal cancer cells. J Hematol Oncol 7: $73,2014$.

28. Mohammed MK, Shao C, Wang J, Wei Q, Wang X, Collier Z, Tang S, Liu H, Zhang F, Huang J, et al: Wnt $/ \beta$-catenin signaling plays an ever-expanding role in stem cell self-renewal, tumorigenesis and cancer chemoresistance. Genes Dis 3: 11-40, 2016. 